Repères sur les systèmes éducatifs

\title{
Les politiques régionales d'éducation en Allemagne depuis PISA
}

\section{Claire Dupuy}

\section{OpenEdition}

\section{Journals}

Édition électronique

URL : http://journals.openedition.org/ries/3581

DOI : 10.4000/ries.3581

ISSN : 2261-4265

\section{Éditeur}

Centre international d'études pédagogiques

Édition imprimée

Date de publication : 1 décembre 2013

Pagination : 18-24

ISBN : 978-2-85420-601-2

ISSN : 1254-4590

\section{Référence électronique}

Claire Dupuy, «Les politiques régionales d'éducation en Allemagne depuis PISA », Revue internationale d'éducation de Sèvres [En ligne], 64 | décembre 2013, mis en ligne le 01 décembre 2015, consulté le 03 mai 2019. URL : http://journals.openedition.org/ries/3581 ; DOI : 10.4000/ries.3581

Ce document a été généré automatiquement le 3 mai 2019.

(c) Tous droits réservés 
Repères sur les systèmes éducatifs

Les politiques régionales d'éducation en Allemagne depuis PISA

\author{
Claire Dupuy
}

1 Depuis la publication, en décembre 2001, des résultats de la première enquête PISA ( Programme for International Student Assessment), l'éducation en Allemagne a suscité d'intenses et larges discussions. La vivacité des débats au sein de l'opinion publique est remarquable car, depuis les années 1960, la réflexion sur les politiques d'éducation était confinée aux cercles des spécialistes de l'éducation. L'ampleur des débats s'explique par le "choc» produit par les données PISA, qui classaient les performances des élèves allemands de 15 ans sous la moyenne des pays de l'OCDE. Mais elle s'explique certainement aussi par le fait que les politiques d'éducation des régions allemandes se trouvent à l'intersection de plusieurs dynamiques politiques et institutionnelles, partiellement contradictoires et qui ont contribué à exacerber les débats. La première dynamique à prendre en compte est le fait que les politiques d'éducation, et plus précisément les politiques d'enseignement secondaire, sont au cœur de la souveraineté des régions allemandes (Kulturhoheit) et que, suite aux évolutions du fédéralisme allemand depuis la fin des années 1960 vers toujours plus d'enchevêtrement des compétences des régions et du niveau fédéral, ces politiques sont devenues l'une des seules dont les Länder ont la responsabilité exclusive. Dans les dernières décennies, et particulièrement lors des réformes récentes du fédéralisme, les régions ont donc tenté de protéger l'enseignement secondaire des tentatives d'intervention du niveau fédéral et de maintenir leur capacité à développer de manière autonome leurs propres politiques d'éducation. La conférence des ministres de l'éducation (Kultusministerkonferenz-KMK), qui rassemble les ministres régionaux de l'éducation, a précisément été créée dans ce but au sortir de la Deuxième Guerre mondiale. Cette organisation a conduit à la coordination horizontale de certains versants des politiques régionales d'éducation, par exemple les dates du début et de fin de l'année scolaire, la reconnaissance des diplômes et les 
exigences pour les diplômes de fin d'étude secondaire. La deuxième dynamique renvoie à la forte politisation partisane des questions d'éducation, qui voient se distinguer, dans les discours et les pratiques, les politiques du parti social-démocrate et celles du parti démocrate-chrétien. Enfin, une troisième dynamique est à prendre en compte, celle des préférences des citoyens allemands. De manière répétée et encore récemment dans le cadre d'une enquête de la Fondation Bertelsmann publiée en 2006, ceux-ci ont exprimé leur souhait de politiques d'éducation uniformes sur l'ensemble du territoire fédéral.

2 Ces trois dynamiques ont nourri les changements majeurs des politiques régionales d'éducation que l'on constate depuis une décennie en Allemagne. Nous allons successivement montrer comment deux des principales évolutions ${ }^{1}$, les réformes de la structure des systèmes scolaires et l'introduction de standards nationaux relatifs aux programmes scolaires, s'expliquent par les interactions entre ces dynamiques politiques et institutionnelles.

\section{Les mobilisations pour le changement des politiques régionales suite à $\mathrm{PISA}$}

3 Suite à la publication des résultats des enquêtes PISA successives, les discussions se sont concentrées sur les solutions pour améliorer la performance des élèves et des systèmes scolaires régionaux. De nombreux acteurs ont participé à ces débats et se sont mobilisés en faveur de l'une ou l'autre de ce qu'ils présentaient comme des solutions.

Le gouvernement fédéral est l'un des principaux acteurs mobilisés. Depuis les années 1960 , et tout particulièrement la fin de cette décennie, il a tenté de gagner des compétences en matière éducative. Les acteurs fédéraux ont mis en avant de manière répétée plusieurs arguments. Le principal, autour desquels s'organisent les autres, est celui selon lequel le gouvernement fédéral serait le seul détenteur de la capacité à assurer et à réaliser les intérêts nationaux, et en particulier l'équivalence des conditions de vie sur le territoire national. C'est dans cette perspective qu'à partir de la publication des résultats de la première enquête PISA, le gouvernement fédéral a défendu une révision générale de l'éducation en Allemagne. Dans de très nombreux articles de presse et déclarations gouvernementales, les résultats de PISA ont été interprétés par la Chancellerie et par le ministère fédéral de l'éducation et de la recherche comme constituant la preuve de la défaillance des régions allemandes à organiser et faire fonctionner des systèmes scolaires performants. L'organisation fédérale de l'éducation a été explicitement désignée par le gouvernement fédéral comme la responsable des mauvais résultats de l'Allemagne et les gouvernements régionaux, dans leur ensemble, ont été jugés coupables de la situation. Pour y remédier, le gouvernement fédéral propose l'instauration d'un cadre national des politiques régionales d'éducation, dont l'adoption conduirait donc à une revalorisation de son rôle dans les questions scolaires.

Les partis politiques et les syndicats enseignants sont aussi parmi les acteurs les plus mobilisés dans ces débats, avec les maires des (petites) villes, les partenaires de coalition, les groupes d'opposition et les acteurs économiques. La question sur laquelle leurs discussions se sont focalisées concernait la performance des systèmes scolaires segmentés de manière ternaire et de ceux où les écoles uniques (Gesamtschulen) étaient plus développées ${ }^{2}$. Ce débat était alimenté par deux résultats, apparemment contradictoires, de l'enquête PISA. La Finlande, où le système scolaire est unitaire, a obtenu les meilleurs 
résultats à l'échelle des pays de l'OCDE, et se caractérise par le système scolaire le moins inégalitaire socialement; tandis que, parmi les régions allemandes, c'est la Bavière, où la segmentation ternaire du système scolaire est particulièrement nette, qui se classe première. Les partisans de la segmentation ternaire des systèmes scolaires, d'une part, et de son changement en un système binaire, d'autre part, puisaient donc leurs arguments dans l'enquête PISA.

\section{L'introduction de standards nationaux relatifs aux programmes scolaires}

6 Face à cette avalanche de critiques et d'incitations extrêmement fortes à changer leurs politiques d'éducation, les régions allemandes ont effectivement changé les versants incriminés de leur politique d'éducation, en instaurant des standards nationaux relatifs aux programmes scolaires et en réformant la structure des systèmes scolaires régionaux. Ces standards portent sur les compétences que les élèves doivent avoir acquis à la fin de chaque année. Ils ont été adoptés à partir de 2004 pour l'ensemble des disciplines et les quatre types d'école. Ces standards viennent compléter des dispositions adoptées par la KMK depuis une trentaine d'années, qui définissent les exigences aux épreuves du baccalauréat et des autres diplômes de fin d'étude secondaire. Ils élargissent la démarche adoptée en l'appliquant non plus uniquement aux attendus de ces deux types d'examen, mais aussi aux programmes de chacun des cycles d'enseignement dans les différents types d'école. Si dès ses premières prises de position sur les résultats de la première enquête PISA en décembre 2001, la KMK a insisté sur la nécessité de garantir et d'améliorer la qualité des systèmes scolaires régionaux, les objectifs d'apprentissage, les procédures d'évaluation et les évaluations de leur application ont été initialement pensés comme devant être définis région par région, en partie à l'échelon des établissements scolaires. Ce n'est que dans un deuxième temps, quelques mois plus tard, que l'introduction de standards éducatifs nationaux est proposée et adoptée par les ministres régionaux de l'éducation. Ces standards définissent « les contenus fondamentaux devant avoir été acquis, ainsi que les compétences transversales devant être maitrisées à la fin d'un cycle d'enseignement donné ». En parallèle, les recommandations pour les examens de fin d'étude sont refondées, notamment pour le baccalauréat (Abitur). En mai 2002, la KMK décide aussi du renforcement de l'évaluation de la réalisation de ces standards, par la mise en place de travaux comparés à l'échelle des écoles, des régions mais aussi du territoire national.

7 Le passage d'objectifs d'apprentissage définis région par région à des standards nationaux définissant, par cycle et par type d'école, les compétences que les élèves doivent avoir acquises, est accompagné d'une défense de l'organisation fédérale de l'éducation par les régions, sous la forme de prises de position très explicites de la présidente de la KMK. Plus précisément, la nature des changements introduits par ces standards reflète aussi la menace institutionnelle à laquelle les régions ont fait face. Les standards nationaux rompent en effet avec plusieurs trajectoires dans lesquelles le fonctionnement de cet organisme de coordination s'était inscrit dans les années 1980 et 1990. La première d'entre elles concerne le fonctionnement même de la KMK. Les années 1990 sont caractérisées par un déclin de son importance dans les politiques scolaires régionales. Les régions y coordonnent alors peu leurs politiques. L'adoption de standards nationaux correspond donc à un effort très important de coordination, pour un organisme qui n'a 
réussi à produire de la coordination dans les vingt années précédentes que sur des points mineurs des politiques régionales. L'effort est d'autant plus important que la coordination des régions s'est faite selon un calendrier très resserré. Dès 2004 en effet, un nombre important d'accords avait déjà été conclu ${ }^{3}$.

La deuxième trajectoire avec laquelle rompt l'adoption par la KMK de standards nationaux concerne le contenu même des standards. Si, depuis les années 1970, la KMK produit des recommandations sur les exigences requises à l'examen du baccalauréat et à l'examen de fin de scolarité en Realschule, ces recommandations portent sur le contenu des enseignements dans les classes concernées. Les standards nationaux adoptés par les régions à partir de 2003 orientent donc bien plus les programmes scolaires que ne le faisaient les recommandations relatives aux examens cités, car ils décrivent les compétences que les élèves doivent avoir acquises, et non uniquement certaines caractéristiques du contenu des enseignements qu'ils doivent avoir suivis. L'autonomie de chacune des régions pour déterminer les programmes scolaires s'en trouve ainsi davantage affectée.

Enfin, l'adoption des standards nationaux par la KMK rompt avec une dernière trajectoire, qui concerne les types d'école et les classes ayant fait l'objet de ses décisions de coordination. Lorsque l'on s'intéresse aux décisions prises par cet organisme depuis sa création selon le type d'école concerné, il apparait clairement que le Gymnasium, en particulier ses dernières classes, celles qui préparent directement à l'examen de l'Abitur, a fait l'objet de la majeure partie d'entre elles. Avant l'introduction de ces standards nationaux, les enseignements en Hauptschule et en Realschule n'avaient que très peu fait l'objet du travail de coordination au sein de la KMK. L'adoption de ces standards nationaux s'est accompagnée de la mise en place d'un organisme d'évaluation, IQB ( Institut zur Qualität-sentwicklung im Bildungssystem), chargé de tester la réalisation de ces standards. Le changement majeur des politiques régionales d'éducation, que l'adoption des standards nationaux introduit trouve donc largement son explication dans les relations intergouvernementales entre les régions et le gouvernement fédéral.

\section{Le passage à la segmentation binaire des systèmes scolaires régionaux}

Le deuxième changement majeur des politiques régionales d'éducation dans la dernière décennie est le passage d'une segmentation ternaire à une segmentation binaire des systèmes scolaires régionaux. Les élèves allemands sont traditionnellement orientés selon leurs aptitudes à la fin du premier cycle d'enseignement secondaire, à 10 ou 11 ans selon les régions, vers un type d'école où ils poursuivent leur formation. Trois types d'école peuvent leur être proposés : Hauptschule, Realschule, Gymnasium, en plus d'un quatrième, la Gesamtschule, qui scolarise tous les élèves ensemble, sans distinction de niveau scolaire. Dans le premier Rapport sur l'éducation publié en 2006, la diversité de structure des systèmes scolaires est soulignée (Avenarius et al., 2003), faisant ainsi écho à des constats plus anciens opérés dès les années 1960. Ce versant crucial des politiques régionales d'éducation n'a jamais été coordonné. L'absence de coordination horizontale s'explique par la très forte polarisation partisane que cette question a suscitée depuis les années 1950, structurée par les vues opposées du parti social-démocrate (SPD) et du parti démocrate-chrétien (CDU). De manière générale, le SPD a plutôt été en faveur de l'unification des systèmes scolaires, c'est-à-dire, dans la version la plus extrême en faveur 
d'une seule école pour scolariser tous les élèves (Gesamtschule) ou dans la version la plus répandue, pour une remise en cause de la segmentation ternaire des systèmes scolaires et son remplacement par une segmentation binaire (fusion de la Hauptschule et de la Realschule, Gymnasium), en parallèle du développement des Gesamtschulen. La CDU a, en revanche, plutôt soutenu la segmentation ternaire des systèmes scolaires, et a été défavorable dans l'ensemble aux Gesamtschulen. Derrière ses prises de position se trouvent des conceptions distinctes, et opposées, du rôle de l'école (Turner, 2011). Tout au long de la deuxième moitié du XXe siècle, la polarisation des prises de position des partis sur cette question a été forte, et a même donné lieu à quelques conflits retentissants qui ont durablement affecté les politiques régionales. La polarisation des positions des deux grands partis a été renforcée à partir du début des années 2000, dans un contexte marqué par la publication des résultats de l'enquête PISA Une partie des débats s'est concentrée sur les performances respectives des systèmes scolaires ternaires et binaires, ainsi que celles des Gesamtschulen.

Dans ce contexte, on constate pourtant que, depuis le début des années 2000, la plupart des régions allemandes ont introduit des réformes substantielles de la structure de leur système scolaire (Wolf \& Knoll, 2011). Si les systèmes régionaux étaient principalement organisés sur la base d'une segmentation ternaire de l'offre de scolarisation, la très grande majorité des régions ont introduit une segmentation binaire où le Gymnasium et un deuxième type d'école (dont le nom est variable d'une région à une autre) côtoient la Gesamtschulen. Seules deux régions ont conservé une stricte segmentation ternaire de leur système scolaire, la Bavière et la Hesse. Trois autres régions maintiennent une segmentation ternaire, mais ont introduit des aménagements qui la sapent sur le long terme: soit en introduisant une forme d'école qui regroupe les enseignements de Hauptschule et de Realschule, comme en Basse-Saxe et en Bade-Wurtemberg, soit en homogénéisant la formation des enseignants, qui est l'un des piliers de la différenciation des trois types d'école, comme en Rhénanie du Nord Westphalie. Les onze autres régions, gouvernées à droite comme à gauche, ont choisi une voie moyenne de la réforme de leur système scolaire, l'instauration de la segmentation binaire, entre le maintien de la segmentation ternaire et l'abrogation de toute segmentation de la formation secondaire.

Comme pour l'introduction des standards nationaux, en dépit de la polarisation partisane sur le sujet, c'est la force des menaces que les acteurs mobilisés, en premier lieu le gouvernement fédéral, ont fait peser sur la distribution des compétences éducatives qui ont conduit la grande majorité des Länder à adopter, de leurs propres gré, les réformes souhaitées par ceux qui argumentaient en faveur du changement de la structure des systèmes scolaires.

En conclusion, les changements des politiques régionales d'éducation qui ont eu lieu dans la dernière décennie semblent paradoxaux. C'est pour maintenir leur capacité à développer de manière autonome leurs politiques d'éducation, et se préserver des tentatives d'intervention du gouvernement fédéral, que les régions en viennent à adopter des réformes majeures similaires d'une région à une autre. 


\section{BIBLIOGRAPHIE}

AVENARIUS H., et al. (Éds.). (2003) : Bildungsbericht für Deutschland : Erste Befunde. Opladen : Leske + Budrich.

DUPUY C. (2012) : La course vers le milieu : Compétition et politiques régionales d'éducation en France et en Allemagne. Canadian Journal of Political Science / Revue canadienne de science politique, 45 (4), 881-907.

TURNER E. (2011) : Political Parties and Public Policy in the German Länder. When Parties Matter. Basingstoke : Palgrave Macmillan.

WOLF F. \& KNOLL C. (2011) : Lehren aus des bundesländervergleichenden Bildungspolitikanalyse : Zehn Thesen im Lichte des Wohlfahrtsstaats. In J. Schmid, K. Amos, J. Schrader \& A. Thiel (Éds.), Welten der Bildung? Vergleichende Analysen von Bildungspolitik und Bildungssystemen (pp. 101-120). Baden-Baden : Nomos.

\section{NOTES}

1. Parmi ces évolutions majeures on compte aussi le passage de la demi-journée d'école à la journée complète (Ganztagschule). Contrairement à celles sur lesquelles cet article se concentre, ce passage a été relativement consensuel. Il a été financé par un programme incitatif du gouvernement fédéral.

2. En Allemagne, les systèmes scolaires régionaux sont traditionnellement structurés autour de trois types d'école, après l'école élémentaire. En Hauptschule, la formation est centrée autour de l'acquisition de savoirs pratiques accompagnés d'une formation générale. Une fois le diplôme de fin d'étude obtenu, les élèves poursuivent leur formation par un apprentissage professionnel. En Realschule, les enseignements sont centrés sur l'acquisition d'une formation générale solide permettant une poursuite d'étude dans des voies professionnelles. Au Gymnasium, les élèves préparent l'Abitur, l'équivalent fonctionnel du baccalauréat, qui leur permet ensuite d'accéder à l'enseignement supérieur. La Gesamtschule est une école où tous les élèves d'une même tranche d'âge sont scolarisés ensemble, indépendamment de leurs aptitudes, et sans être soumis à la sélection qui les oriente, à l'âge de 10 ou 12 ans selon les régions, dans l'un des trois types d'école traditionnels.

3. Les standards nationaux pour la classe 4 en allemand et mathématiques avaient à cette date déjà été adoptés, comme ceux en allemand, mathématiques et première langue vivante (anglais ou français) pour le diplôme de fin de scolarité en Hauptschule, et ceux en allemand, mathématiques, première langue vivante (anglais ou français), chimie, physique et biologie pour le diplôme de fin d'études en Realschule. 
INDEX

Mots-clés : politique éducative, PISA : programme international pour le suivi des acquis des élèves

Index géographique : Allemagne

Keywords : educational policy, PISA: Program for International Student Assessment

Palabras claves : política educacional, PISA: programa para la evaluación internacional de los alumnos

\section{AUTEUR}

\section{CLAIRE DUPUY}

Chercheuse associée au Centre d'études européennes, Sciences Po Paris et au Laboratoire des sciences sociales du politique de l'Institut d'études politiques de Toulouse. Elle est chargée de cours invitée à l'Université catholique de Louvain. 